\title{
Simposio \\ ENFERMEDADES PARASITARIAS EN COMUNIDADES INDÍGENAS
}

\section{Estado actual de las parasitosis intestinales en cuatro comunidades indígenas de Colombia}

\author{
Leidy Puerta, Lina Salazar, Luz Velásquez, Iván Darío Vélez \\ Programa de Estudio y Control de Enfermedades Tropicales, Facultad de Medicina, \\ Universidad de Antioquia, Medellín, Colombia
}

\section{Introducción}

En Colombia hay cerca de 83 etnias indígenas que reúnen algo más de 1'300.000 personas, distribuidas en asentamientos dispersos en áreas rurales de difícil acceso (1). Todas ellas se caracterizan por presentar una alta morbilidad causada por enfermedades diarreicas agudas, enfermedades transmitidas por vectores y parásitos intestinales (2). A pesar de tan grave situación, los programas que velan por la salud de las comunidades indígenas, no disponen de sistemas de vigilancia que permitan evaluar la eficacia de las estrategias que se vienen implementando, con miras a lograr su control y prevención.

En lo que respecta al diseño de sistemas de vigilancia epidemiológica, se viene proponiendo tener presente la diversidad étnica y cultural, porque influyen significativamente en la relación entre las personas y los parásitos. De ahí, la importancia de que cada etnia participe en el diseño de sus planes de control y prevención, partiendo de la información contextualizada sobre sus parásitos. Además, es necesario tener en cuenta que la composición parasitaria de cada comunidad indígena también obedece a factores extrínsecos a la etnia, como aquellos de carácter ambiental: temperatura y régimen de lluvias, abundancia y tipos de vegetación y de ecosistemas acuáticos, entre los más relevantes.

Por lo tanto, la caracterización de los parásitos prevalentes en una comunidad indígena constituye el primer paso hacia la prevención y el control, porque provee la información básica que conduce a la identificación de los factores de riesgo. Ante la falta de información sobre el estado actual de las parasitosis intestinales entre la mayoría de las comunidades indígenas de Colombia, se propone ilustrar esta situación a través del diagnóstico preliminar realizado en tres resguardos de la etnia embera katio, dos ubicados en el municipio de Chigorodó (Antioquia) y el otro en el municipio El Carmen de Atrato (Chocó). Se incluyó, además, un asentamiento de la etnia kogui, en el municipio de Dibulla (La Guajira).

\section{Materiales y métodos}

Para realizar la investigación se solicitó la autorización a los gobernantes de cada comunidad. Las poblaciones embera y kogui están distribuidas en resguardos, los cuales son considerados propiedad colectiva y no enajenable; cada resguardo es administrado por un Cabildo que, a su vez, responde a un Cabildo Mayor como máxima autoridad municipal (3).

Los resguardos de Chigorodó son Chigorodocito y Polines, ambos en la cuenca del rio Chigorodó. El resguardo de El Carmen de Atrato, Ilamado El Dieciocho, se localiza en la cuenca del río Atrato. Aunque distantes entre sí, todos se ubican entre los 90 y $350 \mathrm{~m}$ de altitud, presentan una temperatura promedio de $28^{\circ} \mathrm{C}$ y la vegetación corresponde a bosque húmedo tropical (4-6).

Por su lado, la comunidad kogui visitada está asentada en Umandita, sobre los 479 m de altitud, en la cuenca hidrográfica del río Palomino, en la Sierra Nevada de Santa Marta. La vegetación corresponde a bosque seco tropical $(4,7)$.

Entre octubre de 2010 y junio de 2011 se recolectaron 217 muestras de heces; en cada una de las comunidades se obtuvo una sola muestra por persona. Las muestras se preservaron en formol al $5 \%$ hasta el momento de su análisis. El diagnóstico parasitológico se realizó empleando la técnica de montaje en fresco con solución salina y lugol. Para aumentar la sensibilidad del diagnóstico, se empleó la técnica de sedimentación de Lumbreras.

\section{Resultados}

En el examen coprológico convencional se identificaron quistes y huevos de diferentes 
parásitos; algunos huevos de helmintos se identificaron bajo estereomicroscopio.

La prevalencia de parásitos intestinales fue del $100 \%$ para las muestras analizadas, en las que se identificaron doce especies entre patógenos y comensales. Todas las comunidades comparten ocho especies, las otras cuatro están distribuidas entre las comunidades embera y kogui, cada una con dos especies parasitarias diferentes.

El $85 \%$ de la población presentó poliparasitismo. El promedio de especies parasitarias por individuo fue de tres y seis el número máximo de especies halladas por persona.

Los protozoos patógenos más prevalentes fueron: Blastocystis sp. (73 \%), Entamoeba histolytical dispar (33\%) y Giardia intestinalis (28\%). En cuanto a los helmintos, predominaron Ascaris lumbricoides (46\%) y Trichuris trichiura (40\%).

\section{Discusión}

La alta similitud entre las prevalencias de las especies de parásitos intestinales que se presenta en las comunidades, a pesar de las diferencias culturales y la distancia geográfica, sugiere que la transmisión de protozoos y helmintos está favorecida por el escaso conocimiento sobre los ciclos de vida de estos parásitos, las fuentes de infección y la forma de prevenirlos.

Al comparar los resultados de la presente investigación con los registrados para comunidades de Argentina, Bolivia, México y Venezuela, se observa similitud en la prevalencia de Blastocystis sp., A. lumbricoides y $T$. trichiura (8-12). La presencia del protozoo sugiere que las comunidades no están utilizando métodos eficaces para tratar el agua destinada para el consumo. A su vez, los geohelmintos señalan un manejo de los excrementos que favorece la presencia de huevos en el suelo y el posterior desarrollo larvario.

Por lo tanto, se vuelve imperativo involucrar a la comunidad en la creación de programas adaptados a su realidad cultural y social, ya que los pocos que existen fueron diseñados desde la perspectiva occidental y no han sido exitosos. El reto está en encontrar la forma idónea de comunicación para que esta información llegue a la comunidad afectada, de manera que se pueda sostener un diálogo de saberes. De esta manera, será más fácil para cada etnia identificar las herramientas que desde su acervo cultural se consideren las adecuadas para dar solución al problema.

\section{Bibliografía}

1. Departamento Administrativo Nacional de Estadística. La visibilización estadística de los grupos étnicos colombianos. Bogotá: DANE; 2010.

2. Proyecto de la construcción colectiva hacia la formación de una cultura saludable en comunidades embera de Antioquia, mediante un diálogo de saberes, 2004-2007. Memorias, Congreso Colombiano de Parasitología y Medicina Tropical. Biomédica. 2009; 29.

3. República de Colombia. Constitución Política de Colombia de 1991, artículos 329 y 330. Santafé de Bogotá: Ediciones J. R. Bernal; 1991.

4. Holdridge L. Ecología basada en zonas de vida. San José, Costa Rica: Editorial IICA: 1992.

5. Plan de Salud Territorial, 2008-2011. Chigorodó: Alcaldía Municipal; 2008.

6. Plan de desarrollo municipal, 2008-2011. El Carmen de Atrato: Alcaldía Municipal; 2008.

7. Plan de desarrollo municipal, 2008-2011. Dibulla: Alcaldía Municipal; 2008.

8. Devera R, Finali M, Franceschi G, Gil S, Quintero O. Elevada prevalencia de parasitosis intestinales en indígenas del Estado Delta Amacuro, Venezuela. Biomédica. 2005;25:289-91.

9. Rivero Z, Maldonado A, Bracho A, Gotera J, Atencio $R$. Enteroparásitos en indígenas de la comunidad Japrería, Estado Zulia, Venezuela. Interciencia. 2007;32:270-3.

10. Luna $S$, Jiménez $S$, López R, Soto M. Prevalencia de parasitismo intestinal en niños y mujeres de comunidades indígenas del río Beni. Visión Científica. $2007 ; 1$.

11. Menghi C, luvaroF, Dellacasa M, GattaC. Investigación de parásitos intestinales en una comunidad aborigen de la provincia de Salta. Medicina. 2007;67:705-8.

12. Guevara Y, De Haro I, Cabrera M, García de la Torre G, Salazar-Schettino P. Enteroparasitosis en poblaciones indígenas y mestizas de la Sierra de Nayarit, México. Parasitología Latinoamericana. 2003;58:30-4. 


\title{
Las naciones indígenas originarias de Bolivia frente a la leishmaniasis
}

\author{
Susana Revollo \\ Laboratorio de Ingeniería Genética, Facultad de Ciencias Farmacéuticas y Bioquímicas, \\ Universidad Mayor de San Andrés, La Paz, Bolivia
}

El territorio andino amazónico en los países andinos posee una sorprendente diversidad de ecosistemas, es el resultado de un extraordinario proceso de creación y evolución que se inició hace unos 50 millones de años. Esta geografía es hoy un elemento estratégico porque concentra más del $2 \%$ de la biodiversidad de todo el planeta, el $10 \%$ del agua dulce del mundo y el $35 \%$ de la superficie boscosa de América Latina y el Caribe. La amazonia representa el $59 \%$ de nuestros territorios, juntos representamos el 2,5\% de la superficie terrestre del planeta y aun así somos una de las zonas más ricas en especies de mamíferos, aves, anfibios, mariposas, peces y plantas.

Estas condiciones de diversidad natural han determinado la diversidad étnica y cultural de nuestra región. Habitantes originarios que a partir de su conocimiento, han forjado modos de vida y paisajes. Los recursos biológicos han sido los pilares sobre los que se han levantado nuestras grandes civilizaciones. El territorio andino amazónico boliviano conglomera a sus civilizaciones en 36 naciones indígenas originarias, reconocidas por su Estado Plurinacional. En el altiplano están principalmente las poblaciones aymaras y quechuas mientras que, en la amazonia y el chaco se encuentran el resto de las poblaciones indígenas: araonas, ayoreos, baures, canichanas, cavineños, chacobos, esse ejjas, guarasugwe, sirionós, afrobolivianos, cayubabas, chiquitanos, guarayos, lecos, mosetenes, movimas, moxeños, tapietes, urus, yurucares, joaquinianos, yuques, machineris, yaminahuas, chimanes, tacanas, mores, pacahuaras, reyesanos, weenhayeks, guaranies, nahuas, toromonas e itonamas, cada uno de estos pueblos con su propia identidad, cultura y lengua (1).

Las poblaciones amazónicas de Bolivia experimentaron procesos misionales producto de la colonización durante muchos años donde los pueblos indígenas fueron obligados a convivir con otras poblaciones en medio de la selva poniendo en riesgo su integridad plena $(2,3)$. El despertar de los pueblos originarios a fines del siglo XX se plasmó en el comienzo de una serie de luchas de reivindicación cultural, territorial y, sobre todo, de reconocimiento por el Estado de los pueblos y culturas olvidadas y marginadas desde la creación misma de la República de Bolivia (4).

Bondadosamente, la naturaleza les ha puesto en vida en paraísos celestiales de naturaleza pura, no obstante que de las mismas el $95 \%$ habita en regiones endémicas frente a medios transmisores de las enfermedades tropicales. Existe una historia en torno a estas poblaciones y su propia lucha frente a las enfermedades endémicas de estas regiones, entre las más frecuentes esta la leishmaniosis en sus diferentes formas patológicas, las mismas que representan un problema de salud pública serio $(5,6)$. La leishmaniasis es la mayor causa de enfermedad y morbilidad en Bolivia, donde hay un estimado de 1.800 casos nuevos anualmente (7). Cerca de 21 especies de Leishmania han sido reportadas como causantes de la infección humana (5). La enfermedad es una zoonosis trasmisible que es el resultado del parasitismo de un vertebrado por un protozoo flagelado del género Leishmania (8).

Los estudios epidemiológicos han demostrado la presencia de especies diferentes de Leishmania en la misma región geográfica en Bolivia $(9,10)$. Las especies de Leishmania identificadas en Bolivia son: L. braziliensis (La Paz, Cochabamba, Santa Cruz, Beni, Pando, Tarija), L. chagasi (La Paz, Santa Cruz), L. amazonensis (La Paz, Santa Cruz, Cochabamba), L. lainsoni (La Paz, Cochabamba) y L. guyanensis (Cochabamba). Las poblaciones indígenas originarias son las más afectadas, puesto que el $85 \%$ vive en condiciones precarias de salubridad y están inmersas en medios de ecótopos silvestres donde los insectos transmisores de estas enfermedades satisfacen plenamente sus apetitos cotidianos.

En el año 2007 se registraron 3.253 casos, de los cuales, $1.753(55,6 \%)$ correspondieron al Departamento de La Paz, el mismo que notificada cada año la mayor cantidad de casos que afectaba principalmente a las poblaciones tacanas, chimanes, mosetenes, esse ejjas, reyesanos y lecos. Sin embargo, en Pando, existen municipios con muy alta transmisión, que no se refleja en un número elevado de casos, porque son pocos 
habitantes por pueblos indígenas originarios. En estas regiones, los pueblos más afectados son los machineris, yaminahuas, pacahuaras, nahuas y cavineños. Lo propio sucede con el Departamento de Beni; las poblaciones que habitan estas regiones, baures, canichanas, sirionós, cayubabas, movimas, moxeños, yurucares, joaquinianos, mores e itonamas, son poblaciones representadas por un número bajo de habitantes.

En el cuadro 1 se presenta un resumen de los casos notificados de leishmaniasis en todas sus formas por departamento durante el año 2010.Es importante considerar que los casos notificados en municipios no endémicos, corresponden a casos importados, puntualmente se trata de los Departamentos de Oruro, Potosí y Chuquisaca que no son endémicos en leishmaniasis, puesto que están ubicadas en regiones del altiplano.

Los insectos vectores identificados en Bolivia suman alrededor de 120 especies de flebótomos, de las cuales, cinco fueron formalmente involucradas como vectores de Leishmania: transmisor de $L$. chagasi, Lutzomyia longipalpis; transmisor de $L$.

Cuadro 1. Casos notificados de leishmaniasis en todas sus formas por departamento, Bolivia, 2010.

\begin{tabular}{lcrrr}
\hline Departamento & Mujeres & Hombres & Total & \multicolumn{1}{c}{$\%$} \\
\hline La Paz & 642 & 1.071 & 1.753 & 55,59 \\
Beni & 187 & 387 & 574 & 18,20 \\
Pando & 129 & 287 & 416 & 13,19 \\
Cochabamba & 105 & 236 & 341 & 10,81 \\
Santa Cruz & 15 & 29 & 44 & 1,39 \\
Tarija & 11 & 29 & 40 & 1,26 \\
Oruro & 5 & 4 & 9 & 0,28 \\
Potosí & 2 & 7 & 9 & 0,28 \\
Chuquisaca & 2 & 5 & 7 & 0,22 \\
Total & 1.098 & 2.055 & 3.153 & 100 \\
\hline
\end{tabular}

Fuente: Programa Nacional de Leishmaniasis, Ministerio de Salud y Deportes del Estado Plurinacional de Bolivia braziliensis, Lutzomyia yucumensis, Lutzomyia Ilanosmartinsi, Lutzomyia carrerai carrerai, Lutzomyia nuneztovari anglesi, y transmisor de $L$. amazonensis, Lutzomyia nuneztovari anglesi.

\section{Referencias}

1. Herrera E, Cárdenas C, Terceros, E.. Identidades y Territorios Indígenas: Estrategias identitarias de los tacana y ayoreo frente a la Ley INRA. La Paz: Libro de bolsillo; 2004.

2. Armentia FN. Los indios mosetenos y su lengua. Lafone Quevedo SA, editor. La Paz: ASCA; 1901. p. $145-60$ y 288-306.

3. Cardús J. Las misiones franciscanas entre los infieles de Bolivia. Descripción del estado de ellas en 1883 y 1884. Barcelona. Librería de la Inmaculada Concepción, 1886.

4. Revollo S, Rodríguez N. Las migraciones de los Pueblos Indígenas en la Amazonía Boliviana. Libro original; 2009.

5. Herwaldt BL. Leishmaniasis. Lancet. 1999;354: 1191-9.

6. Murray HW, Berman JD, Davies CR, Saravia NG. Advances in leishmaniasis. Lancet. 2005;366:1561-77.

7. Dirección Nacional de Epidemiología. Proyecto de vigilancia y control de leishmaniasis en los yungas de La Paz. 1997-1998. La Paz, Bolivia; 1996.

8. DesjeuxP. The increase in risk factors for leishmaniasis worldwide. Trans R Soc Trop Med Hyg. 2001;95:23943.

9. Desjeux P, Mollinedo SF, Paredes A, Ugarte G. Cutaneous leishmaniasis in Bolivia. A study of 185 human cases from Alto Beni (La Paz Department). Isolation and isoenzyme characterization of 26 strains of Leishmania braziliensis braziliensis. Trans $\mathrm{R}$ Soc Trop Med Hyg, 1987;81:742-6.

10. Revollo S, Dimier-David L, David C, Lyevre P, Camacho C, Dedet JP. Isoenzyme characterization of Leishmania braziliensis braziliensis isolates obtained from Bolivian and Peruvian patients. Trans R Soc Trop Med Hyg. 1992;86:388-91.

* Proyecto financiado por la cooperación sueca ASDI, 2004-2011

\title{
Desde la mitología kogui hacia un programa intercultural de prevención y control de la enfermedad de Chagas en la Sierra Nevada de Santa Marta
}

\author{
Duámaco Escribano, María Teresa Mojica, Eduino Carbonó, Juan Dib \\ Centro de Investigación en Enfermedades Tropicales-Fundación Salud para el Trópico, \\ Santa Marta, Colombia
}

\section{Introducción}

La tripanosomiasis americana existe en el continente americano desde antes de que se diera el proceso de colonización humana. Pasó de ser una zoonosis a ser una antropozoonosis desde las épocas precolombinas. La transmisión ha dependido de variables económicas, ecológicas y socioculturales que facilitan el contacto entre los vectores, el parásito y el hombre.

La vulnerabilidad de la enfermedad de Chagas al control depende del conocimiento existente sobre su epidemiología y de las herramientas operativas 
disponibles. En la Sierra Nevada de Santa Marta diferentes estudios de seroprevalencia, ecoepidemiología y morbilidad han demostrado que esta parasitosis es altamente endémica en las comunidades indígenas llegando a reportar prevalencias hasta de $89 \%$ en algunos asentamientos.

En estudios previos sobre la implementación e integración de estrategias de control de la enfermedad de Chagas en esta región del país se pudo confirmar que la aplicación de los métodos convencionales de control vectorial mediante las actividades de rociado con insecticidas sintéticos en viviendas infestadas no es una alternativa viable ni culturalmente aceptada. El uso de insecticidas sintéticos requiere de personal técnico capacitado, aspersores costosos y disponibilidad de grandes recursos económicos. El pensamiento ecológico propio de la cosmogonía kogui señala los insecticidas sintéticos como dañinos para los animales y el ambiente.

La ubicación de las viviendas indígenas y los patrones de asentamiento propios de estas poblaciones dificultan el desarrollo de las actividades operativas de control vectorial convencional y aumentan considerablemente sus costos a pesar de contar con personal local capacitado. Además, se ha demostrado la superposición de un ciclo enzoótico y uno doméstico de transmisión vectorial con presencia de Triatoma dimidiata silvestre y Rhodnious prolixus domiciliado en varios asentamientos.

Sin embargo, como resultado de los estudios de conocimientos, actitudes y prácticas de estas poblaciones respecto al vector y los estudios de etnobotánica en esta región del país, se han podido identificar diferentes métodos que podrían orientar nuevas intervenciones de control bajo la conceptualización de la cultura y la epidemiología local.

\section{Objetivos}

Identificar y validar métodos de control vectorial de la enfermedad de Chagas fundamentados en conocimientos y prácticas tradicionales con respecto al vector que permitan implementar una alternativa viable y culturalmente aceptada en las comunidades indígenas de la Sierra Nevada de Santa Marta.

\section{Metodología}

Utilizando herramientas de investigación cualitativa y con entrevistas semiabiertas a la comunidad y varios líderes tradicionales y espirituales de los koguis (mamos) se desarrolló el estudio de la cosmovisión de los koguis con respecto a los insectos transmisores de la enfermedad de Chagas y las prácticas tradicionales indígenas de control vectorial.

Utilizando este conocimiento y la práctica de etnobotánica kogui en el control de insectos, se realizó un estudio experimental mediante el desarrollo de bioensayos en campo que permitieron evaluar la efectividad de métodos tradicionales de control vectorial fundamentados en el uso de insecticidas botánicos y prácticas espirituales.

Para el desarrollo de este estudio se seleccionó un asentamiento kogui con una infestación del $35 \%$. Las viviendas infestadas se dividieron aleatoriamente y mediante un estudio de casos y controles fueron expuestas a métodos de control tradicional indígena. Las muestras de plantas fueron previamente identificadas (Neurolaena lobata, Ricinus communis y Solanum mamosum), recolectadas y confrontadas en el herbario de la Universidad del Magdalena. Las hojas y frutos de Neurolaena lobata, Ricinus communis y Solanum mamosum se prepararon por maceración siguiendo las instrucciones de los autoridades tradicionales indígenas.

Previo al uso de insecticidas vegetales, las viviendas fueron sometidas a rituales tradicionales de pagamento y confieso por parte del líder y sus habitantes (actividades propias del trabajo interno espiritual). Las muestras vegetales maceradas fueron colocadas en un calabazo y luego encendidas en el interior de la vivienda hasta terminar la combustión y el efecto fumígeno. La intervención se realizó una vez al mes y los indicadores entomológicos fueron analizados y comparados antes y después de cada intervención.

\section{Resultados}

La mayoría de los encuestados fueron hombres, debido al poco papel de participación de la mujer indígena en las actividades interculturales.

Cerca de la mitad de los entrevistados respondió haber escuchado de la existencia de la enfermedad de Chagas. El conocimiento sobre la transmisión vectorial (por triatominos) fue mencionada sólo en $2 \%$ de los entrevistados. Respecto a las manifestaciones clínicas de la enfermedad, solamente $7 \%$ de los entrevistados pudieron mencionar alguno de sus signos o síntomas de enfermedad aguda. El trastorno cardíaco no fue mencionado.

El $95 \%$ de los entrevistados demostró conocer los vectores y sus criaderos e indicaron que se alimentan de sangre. El nombre común con que los 
identifican es el de "kuigja". Al preguntar respecto a los criaderos de los triatominos, los techos de palma y las paredes de barro agrietadas fueron los lugares más mencionados. El $80 \%$ atribuyó la nocividad del insecto al hecho de que succionan sangre. Al interrogarlos sobre los métodos utilizados para evitar la presencia de los triatominos en la vivienda, más de la mitad consideró que "el confieso, el pagamento y los trabajos del mamo" son la mejor manera de mantener los vectores alejados de la casa por largos períodos de tiempo. Cerca de la mitad consideró que la eliminación del vector es una responsabilidad personal. Una proporción menor (8\%) indicó que corresponde a los profesionales de la salud (IPS indígena). Solo $3 \%$ encuestados mencionaron que la comunidad debía ser responsable y $10 \%$ no pudieron indicar ningún responsable.

En el estudio experimental el método tradicional de control vectorial, pagamento, confieso e insecticidas botánicos (calabazo fumígeno), demostró una reducción significativa de la infestación y colonización de $R$. prolixus, pero con persistencia de infestación de $T$. dimidiata, probablemente por el comportamiento enzoótico de esta especie.

\section{Discusión}

Aunque cerca de la mitad de los entrevistados dijeron haber escuchado la expresión "enfermedad de Chagas", los mecanismos de transmisión y los síntomas de la enfermedad eran poco conocidos. La relación de los síntomas de la enfermedad con trastornos cardiacos crónicos y que producen incapacidades prácticamente se desconocía en la comunidad. A pesar de que ésta existe como problema verdadero y objetivo, prácticamente es inexistente como hecho cultural. Por lo tanto, las intervenciones de control deben incluir un componente de educación sanitaria intercultural en que se subrayen los mecanismos de transmisión y las manifestaciones clínicas de la enfermedad.

La creencia de que los triatominos son dañinos se relaciona más con su carácter hematófago que con la posibilidad de que transmitan un parásito que causa enfermedad. Para evitar la colonización de las viviendas por el vector se reconoce la importancia del conocimiento de los mayores y los métodos tradicionales. Un programa de control de la enfermedad de Chagas en la Sierra Nevada de Santa Marta debe hacer énfasis en la importancia del autocuidado de la vivienda como medida eficaz y adecuada que no representa mayor costo para la comunidad o el gobierno y que fomenta la participación comunitaria.
Por otro lado, el mayor nivel de conocimientos sobre los hábitos y hábitat del vector en el área facilitan la implementación de un programa de vigilancia entomológica. El contacto personal entre el promotor de salud y los miembros de la comunidad fue la principal fuente de información entre los que mencionaron haber oído hablar de la enfermedad de Chagas. Si se considera que estas comunidades son ágrafas, es lógico esperar que los mensajes educativos escritos (folletos, cartillas, etc.) no tengan mayor impacto. De ahí que en las actividades de control deba hacerse un uso combinado de la comunicación mediante transmisión oral, comunicación interpersonal y medios que puedan ser explicados por el promotor de salud y que, al mismo tiempo, permitan establecer una relación directa y personal con los habitantes de la comunidad.

Debido a que el promotor de salud indígena se ha convertido en el principal interlocutor de la educación sanitaria y la medicina tradicional, se recomienda capacitar periódicamente al personal de salud en cuanto a los aspectos epidemiológicos, entomológicos y clínicos de la enfermedad. El control sostenido de los triatominos requerirá del diseño y la ejecución de programas etnoeducativos a largo plazo encaminados a informar a las poblaciones sobre el riesgo de la enfermedad, así como la promoción de medidas básicas y las recomendaciones para el ordenamiento de la vivienda, peridomicilio, manejo de animales domésticos, uso de insecticidas botánicos, que podrían disminuir el contacto del vector con las personas.

Una estrategia integral de control de vectores de la enfermedad de Chagas en la Sierra Nevada de Santa Marta debería optimizar y racionalizar el uso de recursos y herramientas mejorando la eficacia y la eficiencia, a través de la implementación de métodos sostenibles a largo plazo y ecológicamente adecuados, que reduzcan la dependencia de los insecticidas y protejan a la población de la transmisión vectorial de Trypanosoma cruzi.

El presente estudio sugiere nuevos retos y directrices operativas a nivel individual y colectivo para la implementación de un programa intercultural de control de la enfermedad de Chagas en esta región del país, fundamentado en el fortalecimiento de métodos tradicionales y la apropiación social del conocimiento occidental.

\section{Bibliografía}

1. Agudelo LA, Dib J, Rojas W, Triana O. Epidemiología de la enfermedad de Chagas en una comunidad 
indígena de la Sierra Nevada de Santa Marta, Colombia. Biomédica. 2005;25:113.

2. Dib J, Ariza K., Vélez ID, Agudelo LA. Dispersion and distribution of triatomine species in the Indian communities from the Sierra Nevada of Santa Marta. XVth International Congress for Tropical Medicine and Malaria, 2000, Cartagena. Tropical Health for the New Millenium, International Federation for Tropical Medicine, 2000. v.2. p. 84.

3. Dib, JC, K Ariza, Dwelling Characteristics and Triatomine Colonisation in the Indian communities from the Sierra Nevada of Santa Marta in: XVth International Congress for Tropical Medicine and Malara, 2000, Cartagena. Tropical Health For the New Millenium. International Federation for Tropical Medicine, 2000. v.2. p. 85.

4. Dib J, Rojas W, Bedoya G, Escribano D, Triana O, Tibayrenc M, Muro A. Susceptibilidad Genética a la Infección por Trypanosoma cruzi en dos Grupos Étnicos de una región endémica del Norte de Colombia. Enf Emerg. 2010;2:36.

5. Dib J, Egurrola J, Amaris A, Muro A. Ecoturismo y Enfermedad de Chagas Aguda en el viajero: estudio de nueve casos en países no endémicos. Enf Emerg. 2010;12:72-73.

6. Dib J, Agudelo LA, Vélez ID. Prevalencia de patologías tropicales y factores de riesgo en la comunidad indígena de Bunkwimake, Sierra Nevada de Santa Marta. Duazary. 2006;3:38-44.

7. Dib J. Epidemiología molecular de T. cruzi en el Caribe colombiano (tesis). Medellín: Universidad de Antioquia; 2007.

\title{
Etnografía de la malaria en comunidades indígenas del Caribe colombiano
}

\author{
Juan Dib, Duámaco Escribano, María Teresa Mojica, Eduino Carbonó \\ Centro de Investigación en Enfermedades Tropicales-Fundación Salud Para el Trópico, \\ Santa Marta, Colombia
}

\section{Introducción}

Entre las enfermedades transmitidas porvectores, la malaria es uno de los grandes problemas de salud pública en el mundo ya que representa un gran número de casos de enfermedad y muerte. La malaria afecta principalmente la población rural de áreas con características de selva húmeda tropical.

En Colombia, el aumento en la prevalencia de la enfermedad ha sido alarmante en los pueblos indígenas, favorecido por las condiciones socioculturales, ecológicas, el desplazamiento, las nuevas formas de uso del suelo, la inaccesibilidad geográfica y la falta de servicios de salud. La población indígena en Colombia corresponde a casi el $2 \%$ de la población general y está representada por 84 etnias ubicadas en 408 resguardos y con más de 65 lenguas.
8. Dib J, Agudelo LA, Téllez J, Arboleda S, Rojas W, Triana O. Parasitemia y morbilidad de la enfermedad de Chagas en pacientes de una región endémica. Biomédica. 2005;25:114.

9. Mojica MT, Dib J. Integración de estrategias de control de la enfermedad de Chagas en la Sierra Nevada de Santa Marta. Informe final, proyecto Colciencias; 2010.

10. Mojica MT, Egurrola J, Escribano D, Dib J. Seguimiento clínico y serológico de una cohorte de individuos con enfermedad de Chagas en fase crónica indeterminada sin tratamiento parasitológico. Enf Emerg. 2010;12:43.

11. Rodríguez B, Agudelo LA, Mejía A, Dib J, Solari A, Triana O. Dinámica de transmisión de Trypanosoma cruzi en comunidades indígenas de la Sierra Nevada de Santa Marta, Colombia. Biomédica. 2007;27:214.

12. Parra GJ, Restrepo M, Restrepo BN Domínguez J. Estudio de tripanosomiasis americana en dos poblaciones indígenas de la Sierra Nevada de Santa Marta. CES Med. 2004;18:43-50.

13. Restrepo M, Parra GJ, Restrepo CA. Morbilidad de la enfermedad de Chagas en la Sierra Nevada de Santa Marta. Informe al Ministerio de Salud. Sabaneta: Instituto Colombiano de Medicina Tropical-CES; 2000.

14. Villegas M. Análisis del territorio y patrones de asentamiento en comunidades indígenas de la Sierra Nevada de Santa Marta (tesis). Bogotá: Universidad de los Andes; 2000. 
consecuencia, utilizar con mucha más eficacia los recursos disponibles.

\section{Objetivo}

Identificar la cosmovisión, conocimientos, actitudes y prácticas que con respecto a la malaria tienen las comunidades wayú, kogui y embera, y, a través de diálogos de saberes, confrontarlos con el conocimiento facultativo para encontrar campos comunes que permitan aportar al manejo y control de esta enfermedad en la comunidad.

\section{Metodología}

A través del contacto y previo aval de las organizaciones indígenas, se realizaron reuniones y entrevistas abiertas en las comunidades indígenas wayú, kogui y embera ubicadas en tres ecorregiones del Caribe colombiano donde la malaria es endémica o epidémica.

Con la participación de los promotores indígenas de salud, las autoridades tradicionales y un grupo de profesionales de la Fundación Salud para el Trópico, se utilizaron las herramientas de la investigación cualitativa permitiendo conocer algunos aspectos básicos de la salud y la enfermedad en cada grupo étnico. Se profundizó en los conocimientos, actitudes y prácticas frente a los síndromes febriles analizando el sistema de salud tradicional y el uso de la etnobotánica médica. Del mismo modo se identificaron las necesidades sentidas por malaria de acuerdo con el diagnóstico colectivo de la morbilidad percibida en la comunidad y el problema de la malaria percibido por los prestadores de servicios de salud (IPS) de esta población y autoridades de salud pública regionales.

\section{Resultados}

Según los hallazgos de esta investigación, aunque hay reportes de casos de malaria en estas comunidades indígenas en los últimos años, la malaria es una enfermedad relativamente nueva en las tres etnias estudiadas. La medicina tradicional desconoce la entidad occidental que recibe este nombre, pero reconoce otras entidades, cuyos síntomas clínicos y su causalidad pueden guiarnos a la comprensión de las enfermedades propias de su cultura.

La malaria, desde el punto de vista antropológico tiene un carácter eminentemente cultural, el significado de la enfermedad en cada grupo étnico toma sus variaciones acorde con su cosmovisión, indicando que cada cultura tiene sus propias lecturas y acciones, diferentes formas de pensar y actuar frente a los procesos de salud, bienestar, enfermedad y muerte. Para los embera, la malaria tiene una causalidad social por ruptura de tabúes. En los wayú, la enfermedad obedece a una causalidad múltiple. Por otro lado, los kogui consideran a la malaria como una consecuencia del rompimiento del equilibrio entre él y su entorno por falta de confieso y pagamento. Las tres etnias realizan su tratamiento por medio del sistema médico tradicional y occidental. Sin embargo, a pesar de mantenerse vigente su sistema médico tradicional y complementarse con la medicina occidental, la malaria en los wayú y los embera se presenta de manera endémica y en los koguis se presentan focos epidémicos.

\section{Discusión y conclusiones}

Estudios recientes en el país han señalado que el programa de control de la malaria ha logrado algunos avances significativos, pero que aún persisten debilidades y nuevas amenazas que no le han permitido hacer frente a la enfermedad y su control. En Colombia, la concepción, organización, estructura, financiamiento y operación del programa de malaria es consecuente con el modelo planteado en el SGSSS a través, principalmente, de dos de sus planes de beneficio dirigidas a actividades individuales y colectivas, el POS (Plan Obligatorio de Salud, a nivel individual, y el PIC (Plan de Intervenciones Colectivas) bajo una estructura descentralizada que, en ocasiones, no ha logrado su potencialidad debido las tensiones y dinámicas propias de cada contexto territorial.

En los últimos cuarenta años la tasa de mortalidad por malaria ha disminuido, pero la tasa de morbilidad ha mostrado un constante incremento. A pesar del esfuerzo, no se ha logrado concretar operativamente en lo local para obtener los resultados esperados acorde con la especificidad de la dinámica de transmisión de la malaria por cada ente territorial, probablemente, debido a la fragmentación del programa desde el financiamiento hasta las competencias por entes territoriales y planes de beneficios, pero también a la insuficiente capacidad técnica desarrollada, a la debilidad en la calidad, cantidad y rotación del recurso humano, al conflicto armado, a los desplazamientos y a la poca planeación consecuente.

Por otro lado, a pesar de que los indígenas hacen parte del SGSSS y pertenecen en su mayoría al régimen subsidiado, existe poca evidencia documentada del programa en estas poblaciones y del cumplimiento de las actividades preventivas y de control de la malaria. Los programas de control de la malaria deben asumir enfoques para conocer las dinámicas propias de cada grupo de población, 
considerando las particularidades de cada grupo étnico. Se ha encontrado que la movilización y la comunicación social pueden ser importantes para el fortalecimiento de la gestión interna del programa de la malaria en los resguardos indígenas, pues producen una respuesta social organizada y de adaptación ante la insuficiencia en la capacidad técnica para asumir de manera adecuada las actividades preventivas y curativas en estas poblaciones.

Desde la dimensión estructural, la participación social, entendida como la participación de las comunidades y las instituciones, no ha tenido un buen desarrollo en el país porque su operación ha sido instrumental dado el cumplimiento de los requisitos de ley y la legitimación de procesos. Por ello, se requiere desarrollar en las comunidades indígenas y los demás actores sociales procesos formativos y organizativos conducentes a lograr puntos de vista integrales y compartidos del problema para que, de esta misma manera, el abordaje de la solución sea integral y logre impactar no sólo el daño y los factores de riesgo, sino también sus determinantes. Paralelo a ello, es necesario mejorar el cumplimiento de las diferentes medidas de control con las comunidades indígenas enmarcadas dentro de su vida cotidiana.

Si fortalecemos el soporte social, la probabilidad de éxito de las actividades preventivas aumenta. Es posible lograr que los indígenas mejoren sus conductas con respecto a las actividades de diagnóstico, tratamiento, uso de toldillos, rociado residual y participación comunitaria, y paralelamente, se fortalezcan los métodos tradicionales que han mostrado ser exitosos. El soporte social desde las instituciones se concreta con la propuesta, desarrollo e implementación de herramientas administrativas, gerenciales, políticas y comunicativas con las comunidades indígenas, que apoyen la medida de adaptación en la cual las instituciones puedan responder oportunamente a la dinámica local de transmisión.

Al proponer una metodología de planeación participativa se recogen las diferentes perspectivas del problema y, por lo tanto, se logra un mayor acercamiento a la realidad y las soluciones, de una manera integral y sostenida. Esta estrategia sugerida de soporte social institucional y comunitario indígena para la sostenibilidad de las acciones preventivas y la modificación de determinantes de la malaria, propone entender el pensamiento indígena a través de sus discursos y sus prácticas culturales médicas, desde las connotaciones rituales y espirituales hasta la incorporación de la etnobotánica médica. La comprensión de las conexiones simbólicas de la enfermedad es necesaria para entender la manera de introducir conceptos interculturales, como sería la relación del vector y la necesidad del tratamiento, tendientes al manejo de la enfermedad como un proceso colectivo. El estudio de la etnografía de la malaria posibilita en la comunidad la comprensión del ciclo de transmisión y su importante relación con el vector. La identificación taxonómica, la biología y la distribución de especies vectores deben ser objeto de estudio para el entendimiento de la dinámica de la transmisión y el diseño de programas interculturales de control más adecuados para cada localidad.

\section{Bibliografía}

1. Agudelo C, Corredor A, Valero MV. Assessing options for an innovate malaria control program on de basis of experience with the new Colombian health social security system. Rev Salud Pública. (Bogotá) 2004;6(Suppl.1).

2. Agudelo C. Sistemas de salud y plan de desarrollo: cobertura y equidad. Rev Salud Pública. (Bogotá) 1999;1:117-7.

3. Alvarado BE, Alzate A, Mateus J, Carvajal R. Efectos de una intervención educativa y de participación comunitaria en el control de la malaria en Buenaventura, Colombia. Biomédica. 2006;26:36678.

4. Céspedes J, Jaramillo I, Martínez R, Olaya S, Reynales J, Uribe C, et al. Efectos de la reforma de la seguridad social en salud en Colombia sobre la equidad en el acceso y la utilización de los servicios de salud. Rev Salud Pública. (Bogotá) 2000;2:14564.

5. Green L, Kreuter M. Health promotion planning: An educational and ecological approach. 2nd edition. Mountain View: Mayfield Publishing Company; 1999. p. 32-42.

6. Kroeger A, Ordóñez J, Aviña A. Malaria control reinvented: Health sector reform and strategy development in Colombia. Trop Med Int Health. 2002;7:450.

7. Lipowsky R, Kroeger A, Vásquez M. Sociomedical aspects of malaria control in Colombia. Social Science and Medicine. 1992;34:6.

8. Londoño JL, Frenk J. Pluralismo estructurado: hacia un modelo innovador para la reforma de los sistemas de salud en América Latina, Documento de Trabajo 353. Washinton, D.C.: Banco Interamericano de Desarrollo; 1997

9. Rojas W, Peñaranda F, Echavarría M. Strategies for malaria control in Colombia. Parasitol Today. 1992;8:141-4. 


\title{
Percepción y accesibilidad de comunidades aborígenes al sistema de salud para la vigilancia y el tratamiento de la infección con Trypanosoma cruzi
}

\author{
Sergio Sosa-Estani, Cecilia Touris, Ana Dell'Arcipriete, Lucía Dri, José Brauntein
}

\begin{abstract}
Instituto Nacional de Parasitología Dr. Mario Fatala Chaben, Buenos Aires, Argentina
Centro Nacional de Diagnóstico e Investigación de Endemo-epidemias, Buenos Aires, Argentina

Administración Nacional de Laboratorios e Institutos de Salud Dr. Carlos G. Malbrán, Buenos Aires,

Argentina

Instituto de Efectividad Clínica y Sanitaria, Buenos Aires, Argentina

Consejo Nacional de Investigaciones Científicas y Técnicas, Buenos Aires, Argentina

Atención Primaria de la Salud, Hospital Las Lomitas, Formosa, Argentina
\end{abstract}

\section{Introducción}

La enfermedad de Chagas causada por el Trypanosoma cruzi es una causa importante de morbimortalidad en Latinoamérica. El $90 \%$ de las comunidades aborígenes de Argentina se encuentran en el área endémica de transmisión vectorial de la enfermedad de Chagas. Las medidas adoptadas por el sistema de salud en las localidades de estudio, para la vigilancia y el tratamiento de la enfermedad de Chagas, no habían obtenido hasta el 2006 el impacto esperado en las comunidades aborígenes. Estas comunidades presentaban altos índices de infestación domiciliaria, infección y transmisión congénita de la enfermedad.

\section{Objetivos}

Los objetivos del estudio fueron: a) describir las tasas de infestación en las viviendas de cuatro comunidades aborígenes de Las Lomitas (Región del Gran Chaco), Formosa, Argentina; la tasa de infección en la población y la tasa de transmisión congénita de $T$. cruzi; b) identificar las dificultades en la transferencia del conocimiento para la prevención de la enfermedad de Chagas y los problemas relacionados con el acceso al sistema de salud para indígenas de comunidades wichí y pilagá en Las Lomitas, provincia de Formosa, en 2006-2007.

\section{Metodología}

Se evaluaron entomológicamente el intradomicilio y el peridomicilio para la detección de triatominos, usando metodología hora/hombre en las viviendas de las cuatro localidades seleccionadas.

La población estudiada por serología para detección de la infección fue la población infantil residente en las localidades de estudio, donantes de sangre y mujeres embarazadas que asistieron al Hospital de Las Lomitas y niños nacidos de mujeres infectadas durante el período de estudio. La serología se hizo siguiendo las normas de diagnóstico de Argentina, utilizando dos pruebas serológicas (HAI y ELISA). Para la detección de infección congénita se utilizó la estrategia estandarizada por el Programa Nacional de Chagas mediante la búsqueda de parasitemia en el primer semestre o la detección de anticuerpos por serología a partir de los 10 meses de vida.

Se realizaron entrevistas abiertas a aborígenes de las cuatro comunidades en estudio y observación de los talleres sobre enfermedad de Chagas realizados por el personal del hospital en la comunidad de estudio. El presente protocolo fue aprobado por el Comité de ética independiente del Hospital Dr. F. J. Muñiz de Buenos Aires.

\section{Resultados}

Las tasas de infestación de las 172 viviendas evaluadas, que corresponden al resultado de detección de triatominos luego del rociado con piretroides, alcanzaron el $32 \%$, con variación según las localidades de estudio entre 19,4 y 53,8 $\%$. La prevalencia de infección en 445 personas fue de $17,5 \%$ y en menores de 5 años de edad fue de $8,6 \%(6 / 70)$. La tasa de infección en donantes de sangre alcanzó a 18,6\% y en mujeres embarazadas fue de $29,1 \%$. La tasa de infección considerada congénita en 47 niños nacidos de mujeres infectadas residentes en viviendas bajo vigilancia fue de $17,0 \%$. El estudio mostró, al momento de su inicio, índices indicativos de transmisión vectorial activa, demostrada en la prevalencia de infección en menores de 5 años de edad. La prevalencia de infección según el lugar de residencia fue mayor en áreas rurales. Estas prevalencias fueron superiores en la comunidad wichí, residente en área rural $(31,8$ $\%)$ o periurbana $(30,7 \%)$, cuando se comparó con la comunidad pilagá, residente en área rural $(14,9 \%)$ o periurbana $(7,2 \%)$. Después del control vectorial con insecticidas, la tasa de infestación se redujo a $3,3 \%$ y se mantiene la vigilancia.

Se contrastan las características de los sistemas de salud wichí y pilagá entre sí y con 
las concepciones de la medicina científica y sus prácticas en la zona. Se observa una mayor tasa de infestación e infección en la población wichí que en la población pilagá. Esta diferencia no aparece, en general entre la población residente en áreas rurales y la residente en el área periurbana. Esta observación nos indica que existen características culturales, más que geográficas, entre los factores que favorecen la infestación y consecuentemente la transmisión, los cuales parecen estar ligados a las formas de vida relacionadas con los cuidados de las viviendas. Los grupos wichí que forman parte del presente estudio han tenido un antiguo contacto con las formas criollas de vida y han incorporado la vivienda semipermanente desde hace más de 100 años. No obstante, si comparamos las estrategias interculturales pilagá con las wichí, es notable que en las primeras buscaron incorporar un gran número de pautas culturales "blancas" adaptándolas a las propias, por lo que asumieron, en este contexto, algunas prácticas de vida occidentales. En cambio, los wichí hicieron de la resistencia cultural una estrategia que les ha permitido conservar de una manera más integra y compacta su cultura, pero que no ha sido eficiente en la adaptación a las formas de vida sedentarias.

Sobre las instituciones wichí y pilagá tradicionales y vigentes relativas a la salud y a la enfermedad, es importante destacar que, si bien la mayor parte de los indígenas recurre al sistema de salud oficial para la cura de algunas afecciones, existen especialistas propios, que básicamente son los shamanes.

La información revelada mediante la metodología cuali-cuantitativa demostró que el personal del sistema de salud reconoce dificultades para la comunicación y atención de estas comunidades y no posee estrategias normativas que permita resolverlas. Se desarrollaron herramientas sistematizadas que permiten al sistema de salud: tomar contacto con la perspectiva aborigen sobre el proceso salud-enfermedad-atención; conocer sus características, y determinar acciones que promuevan un encuentro intercultural que se refleje en una mayor accesibilidad de las comunidades aborígenes al sistema.

La principal diferencia entre las comunidades estudiadas reside en que las categorías pilagá sobre enfermedad pueden integrar fácilmente las nociones médicas occidentales, en tanto que las categorías wichí las enfrentan en bloque, colocándolas en una esfera separada: enfermedades "nuevas" frente a "viejas".
En cambio, en lo que respecta a los aspectos comunes de la situación de ambos pueblos frente a la enfermedad, puede afirmarse de un modo general que:

1. la enfermedad de Chagas está integrado de un modo periférico dentro del sistema de categorías indígenas de la salud y la enfermedad;

2. la integración de esta enfermedad en el conjunto de los sistemas de categorías indígenas es absolutamente necesaria si se desea que esas poblaciones respondan de un modo positivo a las necesidades epidemiológicas que plantea el sistema público de salud;

3. buena parte de la fractura de la comunicación existente entre el Sistema de Salud y la población, se deriva del desconocimiento por parte de los profesionales de la salud de aspectos mínimos relativos a las culturas de los pacientes que tratan, lo cual suele traducirse en actitudes que distancian a los integrantes de las comunidades aborígenes.

\section{Discusión}

Las elevadas tasas de prevalencia de infección en donantes de sangre y en mujeres embarazadas, demuestran también una intensa transmisión en el pasado.

La mayor tasa de infestación e infección en la población wichi comparada con la pilagá, y la ausencia de estas diferencias según lugar de residencia rural o urbana, sugieren que las características culturales más que las geográficas son determinantes que favorecen la infestación, y consecuentemente, la transmisión de la infección en el área de estudio.

En las cuatro localidades la disminución de la tasa de infestación muestra el impacto de las acciones de control del sistema de APS que mantiene las acciones de vigilancia.

El sistema de salud local incorporó procedimientos de prevención primaria y secundaria para evitar nuevos casos e instaurar el tratamiento de la población infectada.

Los resultados advirtieron sobre la necesidad de una revisión de los métodos y procedimientos oficiales de comunicación utilizados para abordar las acciones de control de la enfermedad de Chagas entre las comunidades indígenas actualmente en práctica.

Financiamiento. Este estudio recibió apoyo financiero parcialmente del Programa Especial PNUD/ Banco Mundial/OMS de Investigaciones y Enseñanzas sobre Enfermedades Tropicales (TDR); Becas Ramón Carrillo-Arturo Oñativia 2006, 
Comisión Nacional Salud Investiga, Ministerio de Salud de Argentina, ANLIS Dr. Carlos G. Malbrán.

\section{Referencias}

1. Duarte MB. Medicina occidental y otras alternativas: ¿es posible la complementariedad? Reflexiones conceptuales. Cad Saúde Pública. 2003;19:109-18.

2. Gurtler RE, Segura EL, Cohen JE. Congenital transmision of Trypanosoma cruzi infection in Argentina. Emerg Infect Dis. 2003;9:29-32.

3. Lerin S. Interculturalidad en salud: un reto para la antropología aplicada. México. Centro de Investigaciones y Estudios Superiores en Antropología Social: 2005.

4. Ministerio de Salud, República de Argentina. Guías para la atención al paciente infectado con Trypanosoma cruzi (enfermedad de Chagas).
Disponible en: http://www.msal.gov.ar/chagas/home. htm.

5. Palmer J. La buena voluntad wichí: una espiritualidad indígena. Primera edición. Formosa: APCD; 2005. p. 432.

6. Segura EL, Sosa Estani S, Esquivel ML, Gómez A, Salomón OD. Control of the transmission of Trypanosoma cruzi in Argentina 1999. Medicina (Buenos Aires) 1999;59(Suppl.2):91-6.

7. Sosa-Estani S, Dri L, Touris C, Abalde S, Dell'arciprete A, Braunstein J. Vectorial and congenital tranmisssion of Trypanosoma cruzi in Las Lomitas, Formosa. Medicina (Buenos Aires). 2009;69:424-30.

8. Zidemberg M, Spillmann C, Carrizo R. Control de Chagas en la Argentina. Su evolución. Rev Arg Cardiol. 2004;72:375-80. 